\title{
Influenza vaccination coverage across ethnic groups in Canada
}

\author{
Susan Quach MSc, Jemila S. Hamid PhD, Jennifer A. Pereira PhD, Christine L. Heidebrecht MSc, \\ Shelley L. Deeks MD MHSc, Natasha S. Crowcroft MD MSc, Sherman D. Quan MSc, Stephanie Brien MSc, \\ Jeffrey C. Kwong MD MSc; for the Public Health Agency of Canada/Canadian Institutes of Health Research \\ Influenza Research Network Vaccine Coverage Theme Group
}

See related commentary by Gessner on page 1661 and at www.cmaj.ca/lookup/doi/10.1503/cmaj.120775

\begin{abstract}
Background: The success of influenza vaccination campaigns may be suboptimal if subgroups of the population face unique barriers or have misconceptions about vaccination. We conducted a national study to estimate influenza vaccine coverage across 12 ethnic groups in Canada to assess the presence of ethnic disparities.
\end{abstract}

Methods: We pooled responses to the Canadian Community Health Survey between 2003 and 2009 ( $n=437$ 488). We estimated ethnicity-specific self-reported influenza vaccine coverage for the overall population, for people aged 65 years and older, and for people aged 12-64 years with and without chronic conditions. We used weighted logistic regression models to examine the association between ethnicity and influenza vaccination, adjusting for sociodemographic factors and health status.

Results: Influenza vaccination coverage ranged from $25 \%$ to $41 \%$ across ethnic groups. After adjusting for sociodemographic factors and health status for people aged 12 years and older, all ethnic groups were more likely to have received a vaccination against influenza than people who self-identified as white, with the exception of those who self-identified as black (odds ratio [OR] 1.01, 95\% confidence interval $[\mathrm{Cl}]$ 0.88-1.15). Compared with white Canadians, Canadians of Filipino (OR 2.00, 95\% $\mathrm{Cl}$ 1.67-2.40) and Southeast Asian (OR 1.66, $95 \% \mathrm{Cl} 1.36-2.03)$ descent had the greatest likelihood of having received vaccination against influenza.

Interpretation: Influenza vaccine coverage in Canada varies by ethnicity. Black and white Canadians have the lowest uptake of influenza vaccine of the ethnic groups represented in our study. Further research is needed to understand the facilitators, barriers and misconceptions relating to vaccination that exist across ethnic groups, and to identify promotional strategies that may improve uptake among black and white Canadians.
A ccurate and reliable data on vaccine coverage are essential for evaluating the success of influenza vaccination campaigns. Identifying populations with suboptimal coverage can help program planners design effective, targeted health-promotion strategies. Vaccine coverage has traditionally been described by age and sex; ${ }^{1}$ little research in Canada has examined coverage by ethnicity.

Many studies in the United States have shown large ethnic disparities (> 15\%) in coverage between people who self-identify as white, black or Hispanic among adults with high-risk conditions, ${ }^{2}$ older adults ${ }^{3,4}$ and Medicare beneficiaries. ${ }^{5}$ The reasons for these disparities are complex and may be related to attitudes and knowledge about vaccination, access to health care, socioeconomic status and provider bias. ${ }^{6}$ However, these findings cannot be applied to the Canadian population, which is distinct in terms of the delivery of influenza vaccinations and ethnic composition. Since the late 1970s, the number of non-European immigrants to Canada has risen, contributing to a substantial increase in the visible minority population, from $4.7 \%$ of the total population in 1981 to $16.2 \%$ in $2006 .^{7}$ More than 200 different ethnic origins were reported in the 2006 Census, ${ }^{8}$ with people of South Asian $(4.0 \%)$, Chinese $(3.9 \%)$ and black $(2.5 \%)$ heritage representing the largest proportions of the population.

Canada's National Advisory Committee on Immunization recommends vaccination against influenza for people at high risk of serious infection and their contacts. ${ }^{9}$ The largest risk groups are adults 65 years of age and older and people with certain chronic medical conditions (e.g., cardiac and pulmonary disorders, diabetes,
Competing interests: Jennifer Pereira has been a consultant for GlaxoSmithKline for non-influenzarelated work. No other competing interests were declared.

This article has been peer reviewed.

Correspondence to: Jeffrey C. Kwong, jeff.kwong@utoronto.ca

CMAJ 2012. DOI:10.1503 /cmaj.111628 
cancer, immune-compromising conditions, renal disease, anemia and obesity). Every province and territory provides free influenza vaccinations to these priority groups. Since 2000, Ontario has provided publicly funded influenza vaccinations to all people older than 6 months of age. Other jurisdictions (Alberta, Manitoba, Saskatchewan, Nova Scotia, Nunavut, Yukon and Northwest Territories) have subsequently adopted similar programs. ${ }^{10}$ Although these initiatives should reduce financial barriers to vaccination, there may be other barriers or misconceptions unique to specific groups that affect behaviour.

The purpose of this study was to estimate influenza vaccine coverage across 12 ethnic groups in Canada to assess possible disparities.

\section{Methods}

\section{Survey sample}

We conducted a cross-sectional study using nationally representative data from the 2003, 2005, 2007, 2008 and 2009 cycles of the Canadian Community Heath Survey. ${ }^{11}$ This voluntary survey by Statistics Canada collects information about health status, use of health care and health determinants from people aged 12 years and older. The survey uses a multistage stratified cluster design that samples $98 \%$ of the population, excluding people living on Aboriginal reserves or Crown land, regular members of the Canadian Forces, and residents of institutions (e.g., long-term care facilities, prisons) and certain remote regions.

We combined 5 cycles to increase the size of our sample of ethnic groups to improve the reliability of our estimates. We considered factors such as changes in the content of the survey or its method of delivery, coverage and geography before combining cycles. ${ }^{12,13}$ The response rate across cycles ranged from $73 \%$ to $81 \% .{ }^{11}$

This study received approval from the Health Sciences Research Ethics Board of the University of Toronto.

\section{Outcome measures}

Our dependent variable was self-reported in fluenza vaccination, which we determined with responses to the questions "Have you ever had a flu shot?" and "When did you have your last flu shot?" We considered only those respondents who reported having had a flu shot within the past 12 months to be actively immunized against influenza.

We determined ethnicity, our primary independent variable, with the question "People living in Canada come from many different cultural and racial backgrounds. Are you ... ?" The following choices of response were provided: white, Chinese, South Asian (e.g., Indian, Pakistani, Sri Lankan), black, Filipino, Latin American, Southeast Asian (e.g., Cambodian, Indonesian, Laotian, Vietnamese), Arab (e.g., Egyptian, Lebanese), West Asian (e.g., Afghan, Iranian), Japanese, Korean, Aboriginal (North American Indian [i.e., First Nations], Métis, Inuit) and other. Respondents who selected multiple categories were classified as multiracial, unless one of the categories they selected was Aboriginal. For this reason, some people in the Aboriginal group may have multiracial ancestry. We combined the West Asian and Arab groups owing to the small numbers of people who self-identified as belonging to them. In addition, we grouped together those respondents who self-identified as multiracial and other.

Potential confounders included age, sex, survey cycle, use of health services, immigration status, percentage of years spent in Canada (number of years in Canada divided by age), household income, education, self-perceived health status, living in a jurisdiction with a universal influenza vaccination program during all or most of the study period (i.e., Ontario, Northwest Territories and Nunavut), having a regular doctor, living in a rural area (population concentration < 1000 and population density < 400/ $\mathrm{km}^{2}$ ), marital status, smoking status, ability to speak English or French, and the presence of at least 1 of the following self-reported chronic diseases: asthma, chronic obstructive pulmonary disease (chronic bronchitis or emphysema), diabetes, heart disease, cancer or stroke.

\section{Statistical analysis}

We pooled individual-level responses from multiple survey cycles; the final dataset was considered a sample from an average population observed over all survey cycles. ${ }^{13}$ We weighted all of our analyses to account for the survey's complex sampling design. We calculated variance estimates and coefficients of variation using bootstrapping methods. ${ }^{14,15}$

We estimated ethnicity-specific influenza vaccine coverage for the overall population, for people aged 65 years and older, and for people aged 12-64 years with and without chronic conditions. We also estimated coverage stratified by the presence of a universal influenza vaccination program throughout most of the study period.

We used weighted logistic regression models to determine the association between ethnicity and vaccination, adjusting for potential confounders. We modelled the individual-level odds of influenza immunization, using the $\chi^{2}$ test to 
determine the significance of ethnicity in the adjusted model. To guide the regression analysis, we started with univariate models for each of the factors identified as important predictors from previous studies. ${ }^{1,2,5,16,17}$ We then investigated the correlation matrix, consisting of pairwise correlations between variables, using Spearman rank (for categorical variables) and Pearson product-moment correlations. We tested highly correlated variables in and out of the saturated model to determine their impact on parameter estimates and on the Akaike information criterion, which is used to assess goodness of fit. To minimize the potential for multicollinearity and model overfitting, we removed variables that were highly correlated or resulted in complete separation of data. Finally, we examined linearity among continuous variables to determine whether higher-order terms were more appropriate for modelling. We conducted sensitivity analyses on several subpopulation models (immigrants, people aged 65 years and older, ethnic minorities).

\section{Results}

After excluding respondents for whom data on ethnicity or vaccination were missing ( $n=$ 24670 ), our total sample was 437488 people, which we weighted to represent a Canadian population of 26.0 million people aged 12 years and older. People belonging to ethnic minority groups comprised $18 \%$ of the sample. The sociodemographic characteristics of the sample, by ethnicity, are presented in Table 1.

Among people 12 years of age and older, Canadians of Filipino (41\%, 95\% confidence interval [CI] 37-44), Japanese $(38 \%, 95 \%$ CI $32-44)$ and Chinese $(35 \%, 95 \%$ CI 33-36) descent showed significantly higher influenza vaccination coverage than white Canadians (32\%, 95\% CI 31-32) (Figure 1). Latin American $(26 \%, 95 \%$ CI $23-29)$, West Asian/Arab $(26 \%, 95 \%$ CI 23-29) and black Canadians (27\%, 95\% CI 25-29) had significantly lower levels of coverage (Figure 1). Among people aged 65 years and older $(n=93982)$, coverage was highest among Canadians of Chinese (78\%, 95\% CI 74-83), Southeast Asian (80\%, 95\% CI 68-89) and Japanese (80\%, 95\% CI 71-87) descent, compared with white Canadians (68\%, 95\% CI 68-69) (Figure 1). The West Asian/Arab $(46 \%, 95 \%$ CI $34-63)$ and black $(59 \%, 95 \%$ CI 50-66) groups showed significantly lower coverage levels than the white group (Figure 1).

Among respondents with no chronic disease $(n=285953)$, with the exception of people of Filipino and Japanese heritage, coverage was significantly higher for each ethnic group in jurisdictions with a universal influenza vaccination program (Figure 2). Furthermore, Filipino, Southeast Asian and Chinese Canadians who had no chronic diseases had significantly higher vaccine coverage than white Canadians. For Canadians aged 12-64 years with a chronic disease $(n=$ 56 697), coverage ranged from 34\% (95\% CI $29-40$ ) to $50 \%$ (95\% CI 31-70).

Univariate analysis showed that 5 ethnic groups (black, West Asian/Arab, Latin American, Aboriginal and multiracial/other) were significantly less likely to receive the influenza vaccination than the white group; 3 ethnic groups (Filipino, Japanese, and Chinese) were more likely to receive the vaccination (Figure 3 ). However, after adjusting the model for sociodemographic characteristics, all ethnic groups were more likely to receive the vaccination than were white Canadians, with the exception of people who self-identified as black (adjusted odds ratio [OR] 1.01, 95\% CI 0.88-1.15) (Figure 4). Compared with white Canadians, Filipino (OR 2.00, 95\% CI 1.67-2.40) and Southeast Asian Canadians (OR 1.66, 95\% CI 1.36-2.03) were the most likely to get the influenza vaccination; although still statistically significant, people in the West Asian/Arab (OR 1.22, 95\% CI 1.001.47), Latin American (OR 1.24, 95\% CI 1.001.52) and multiracial/other (OR $1.19,95 \%$ CI 1.05-1.35) groups had only slightly higher odds of getting the vaccination than people in the white group. Health status and several sociodemographic characteristics were also independently associated with vaccination in the univariate and multivariate analyses (Figures 3 and 4).

\section{Interpretation}

Our results show that all ethnic groups, with the exception of black Canadians, had significantly higher uptake of influenza vaccination than white Canadians. Variations in coverage levels persisted even after adjusting for other determinants of vaccine uptake, which suggests that there may be unique barriers and misconceptions influencing these groups differently.

Two recent systematic reviews that examined factors associated with vaccination for $2009 \mathrm{~A}$ (H1N1) pandemic influenza found that ethnicity was associated with vaccine uptake in the United Kingdom (UK), Australia and the US..$^{18,19}$ Furthermore, our results concur with those from other studies that showed higher uptake of childhood vaccinations among certain ethnic populations. ${ }^{20,21}$ In a UK study involving children aged 18 months to 3 years, Mixer and colleagues found that Asian children were more likely to 


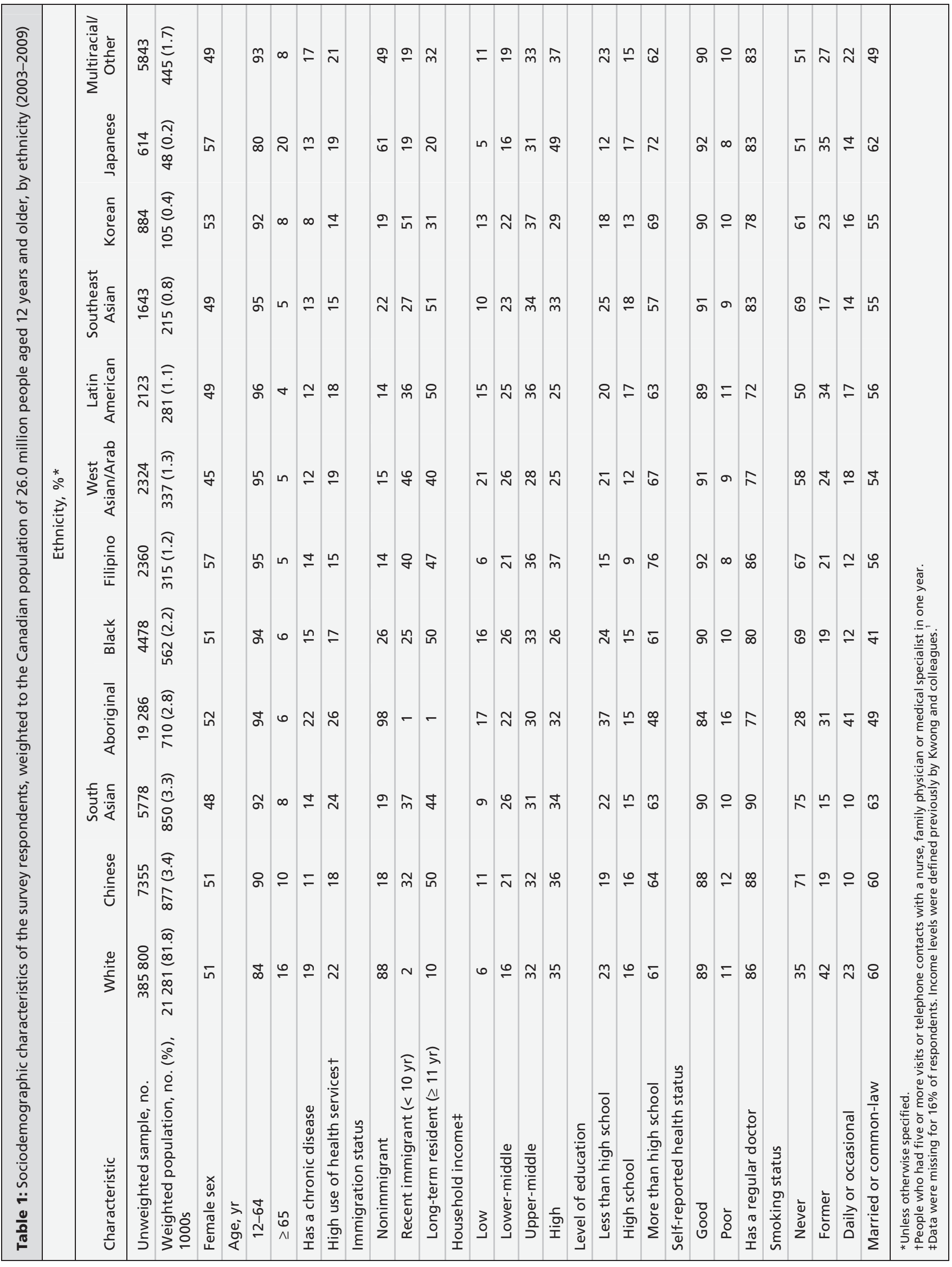


receive the measles-mumps-rubella (MMR) vaccine than black or white children. ${ }^{21}$ These differences were attributed to varying levels of exposure to mass media on the controversy surrounding the MMR vaccine, as well as the influence of family and health professionals on decisions about vaccinations. In addition, the authors suggested that "shielding" from negative media coverage, owing to language barriers and different levels of integration into British society, may have contributed to higher vaccine coverage in certain populations.

Our results could be related to the level of exposure to and support for antivaccination media, because it is unknown whether certain groups in Canada are more likely to be exposed

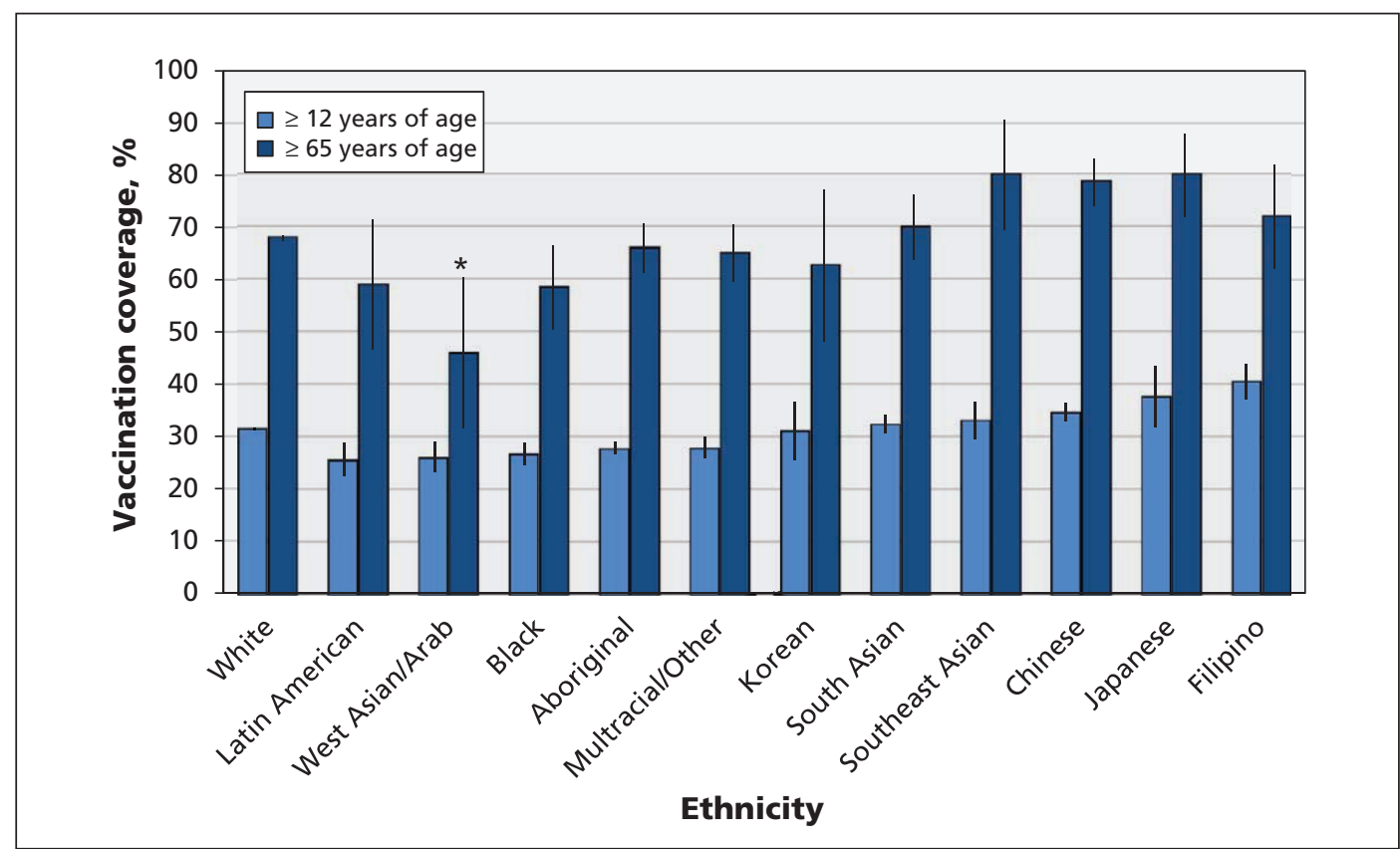

Figure 1: Influenza vaccine coverage, by ethnic group and age, in Canada (2003-2009). Error bars represent $\mathbf{9 5 \%}$ confidence intervals. *A marginal coefficient of variation suggests high sampling variability is associated with this estimate.

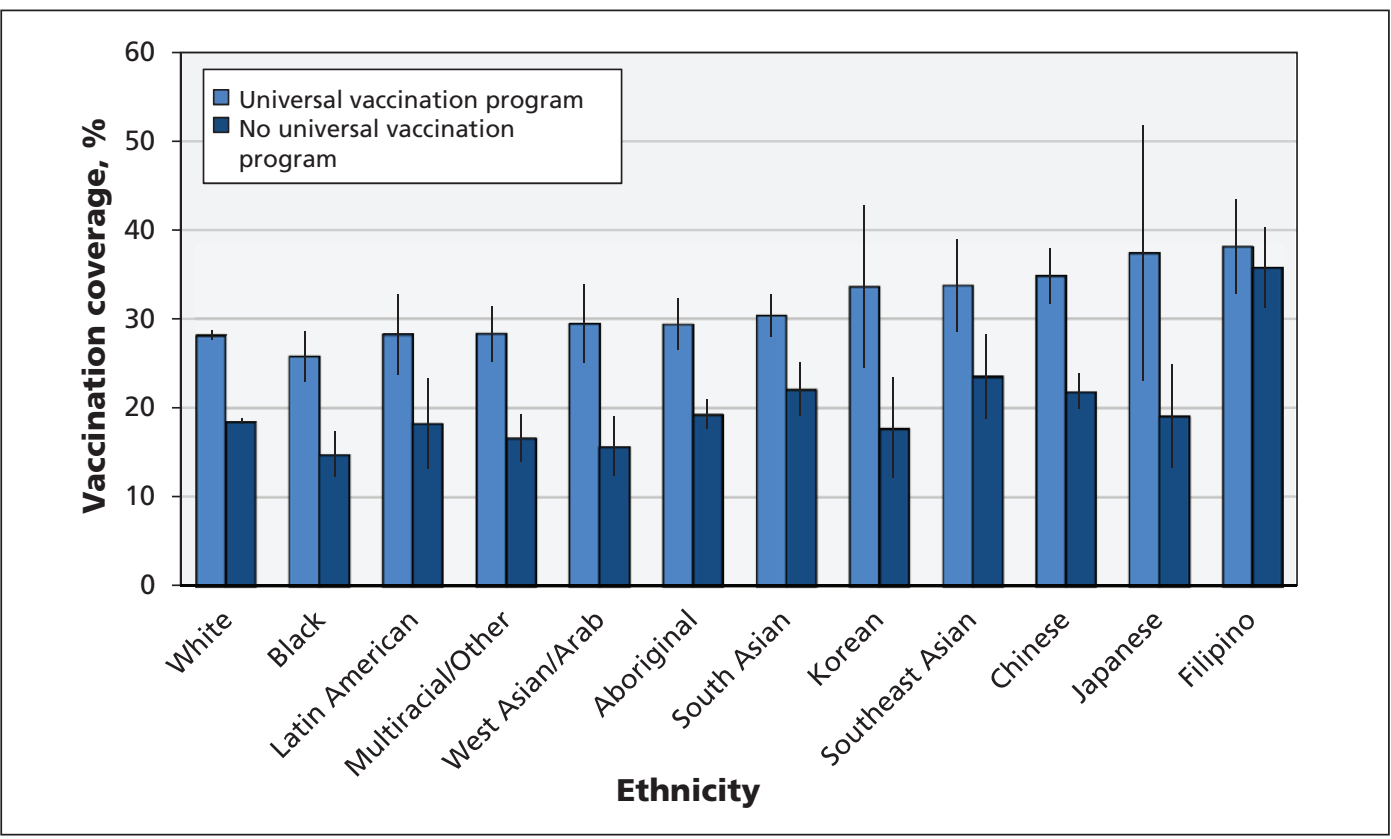

Figure 2: Influenza vaccine coverage, by ethnic group and availability of a universal influenza vaccination program, in Canadians aged 12-64 years without chronic diseases (2003-2009). Error bars represent 95\% confidence intervals. 
to, or supportive of, such media. Further research is needed to understand exposure to antivaccination media by ethnicity, and whether this affects willingness to receive vaccinations.
Our results are similar to those of American studies that have documented ethnic differences in influenza vaccination among Asian populations..$^{2-4,16,22,23}$ The 2008 Behavioural Risk Factor

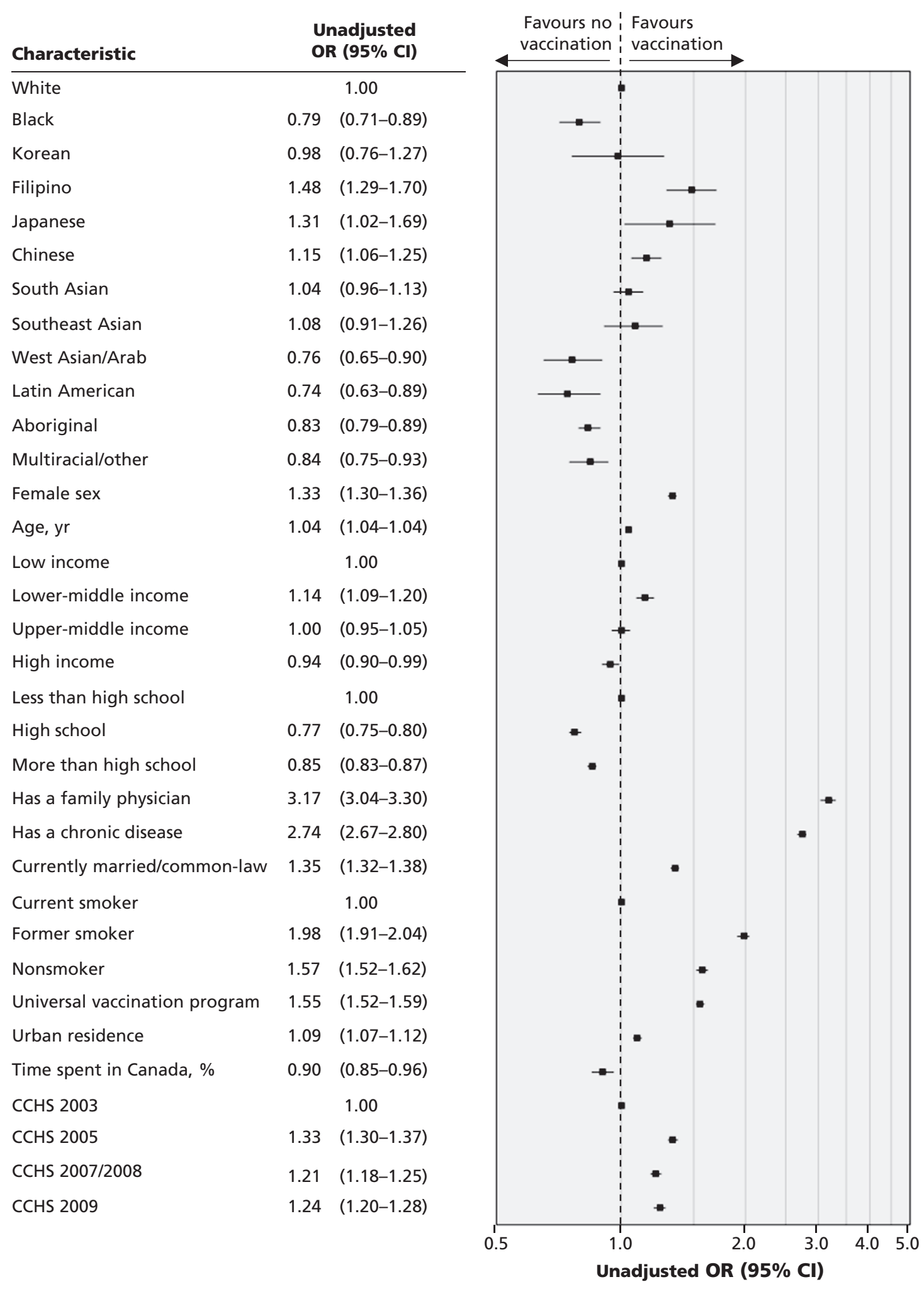

Figure 3: Unadjusted odds ratios for influenza vaccination by ethnicity, sociodemographic characteristic and health status for Canadians aged 12 years and older (2003-2009). CCHS = Canadian Community Health Survey, Cl = confidence interval, OR = odds ratio. 
Surveillance Survey showed that influenza vaccination among Asian Americans aged 50 years or more was significantly higher than among peo- ple from the non-Hispanic white, non-Hispanic black and Native American groups, after adjusting for other variables. ${ }^{4}$ The reasons for this

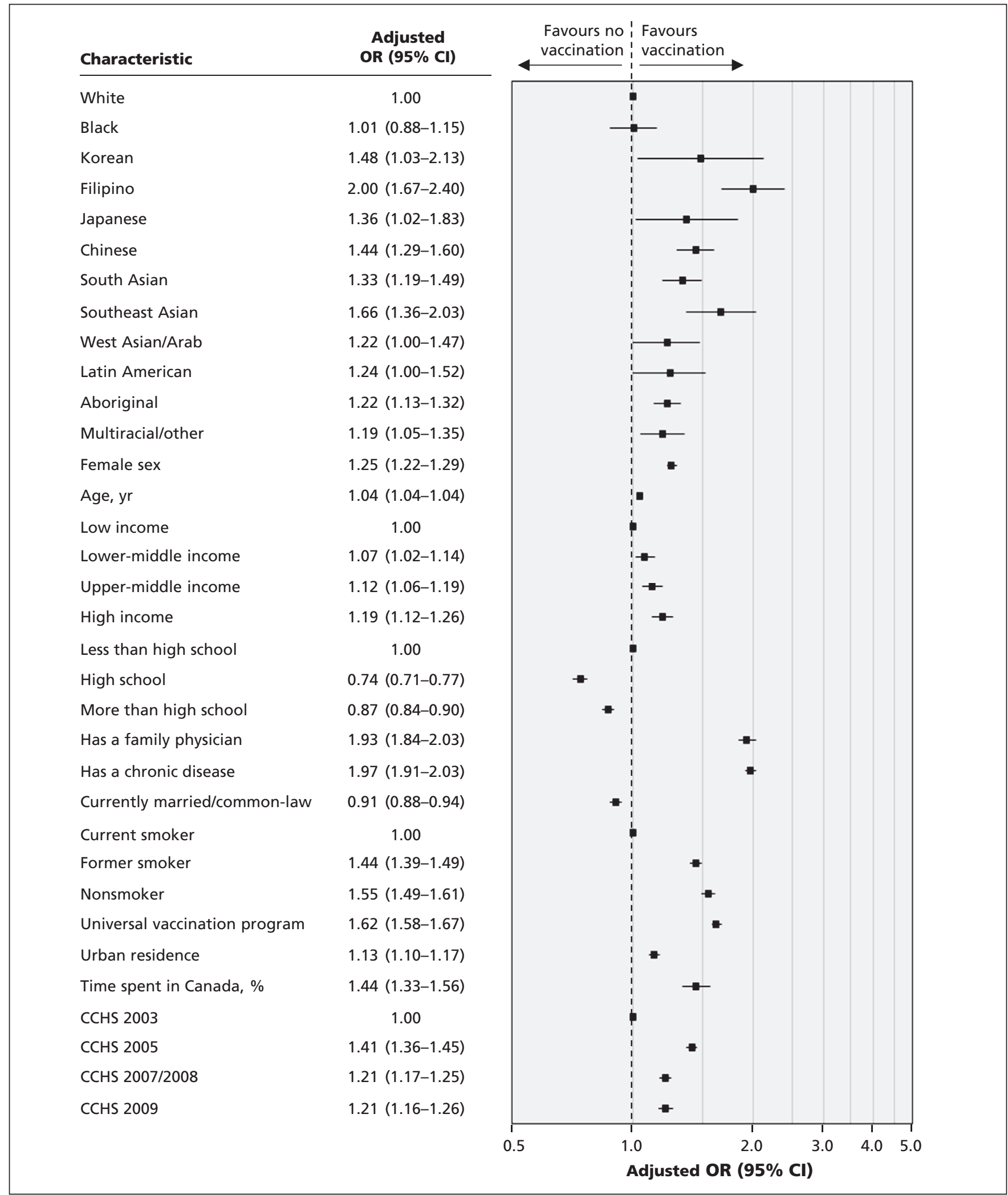

Figure 4: Adjusted odds ratios for influenza vaccination by ethnicity, sociodemographic characteristic and health status for Canadians aged 12 years and older (2003-2009). CCHS = Canadian Community Health Survey, $\mathrm{Cl}=$ confidence interval, OR = odds ratio. 
higher rate of coverage among Asian Americans are not well studied.

A recent survey involving students and staff at an Australian university found that, compared with participants from other regions, Asian-born respondents were significantly more likely to have anxiety concerning the 2009 A (H1N1) influenza pandemic, rate the situation as serious and change specific behaviours to comply with public health measures. ${ }^{24}$ During the past century, 2 influenza pandemics and the severe acute respiratory syndrome coronavirus have originated in Asia; the increased media exposure and precautionary actions resulting from these health events may have heightened awareness among Asian populations. In addition, the psychological impact on people who directly or indirectly experienced negative events as a result of these outbreaks may provide additional motivation to receive vaccinations. Furthermore, people who lived through both of the most recent influenza pandemics that originated in Asia would be at least 50 years old; in our study, the range of coverage among elderly participants was more than twice that of the respondents aged 12-64 years (i.e., $31 \%$ v. $14 \%$ ). Similarly, estimates of coverage among Asian Americans aged 18-64 years and at high risk of infection with the influenza virus were close to the US national average for the 2006-2007 influenza season, but higher coverage was seen among Asian Americans aged 65 years and older. ${ }^{17}$

\section{Limitations}

Currently, there is no efficient method to routinely assess ethnic disparities in influenza vaccination in Canada, because ethnic identifiers are generally not collected on vaccination records. Relying on data from the CCHS does not permit regional or annual estimates, because sample sizes are restrictive among ethnic minority groups.

Self-reported vaccination status is subject to response and recall biases, resulting in over- or underestimates of coverage. However, previous studies have validated self-reported influenza vaccination status. ${ }^{25-27}$

Ethnic categories were based on those of the CCHS questionnaire, and some groups were heterogeneous (e.g., black, white, South Asian, Latin American, multiracial/other). In an effort to avoid exacerbating this heterogeneity, we refrained from combining East Asian or Southeast Asian groups to assess the variability in coverage between these groups, given the pre-existing evidence of disparities..$^{16}$

It was not possible to distinguish people identifying solely as Aboriginal in the CCHS data because Aboriginal people were not asked to state whether they also belonged to other ethnic groups. Therefore, some of the people in the self-identified Aboriginal group included in our study may be of multiracial heritage.

We did not control for status as a health care worker in our analysis, but we are unsure if this would have a significant impact on our results given that less than $7 \%$ of the population work in health occupations, and the proportions of people working in health-related occupations are similar between immigrants and nonimmigrants. ${ }^{28}$

Finally, although the CCHS is conducted in multiple languages, people with language difficulties may be less likely to participate. Because these people may have reduced access to information about vaccinations, coverage among ethnic minorities in this sample may have been overestimated. Language issues may also contribute to a higher response rate among the Aboriginal and white populations compared with other groups, resulting in a larger sample of both groups in this survey.

\section{Conclusion}

All ethnic groups in Canada, with the exception of black Canadians, had higher uptake of influenza vaccination than white Canadians. These differences remained significant even after controlling for sociodemographic characteristics and health status. These results suggest a public health concern that requires further validation and study to determine whether there are barriers to vaccination that are unique to black and white Canadians.

Our findings should not understate the importance of tackling issues of sociodemographics and access to health care that influence uptake and extend across all ethnic groups in Canada. To ensure optimal vaccine coverage, it will be important for public health to work with health care providers, clients and community-based organizations to understand the unique challenges and barriers that affect their communities, and to design appropriate interventions for different populations.

\section{References}

1. Kwong JC, Rosella LC, Johansen H. Trends in influenza vaccination in Canada 1996/1997 to 2005. Ottawa (ON): Statistics Canada; 2007.

2. Egede LE, Zheng D. Racial/ethnic differences in influenza vaccination coverage in high-risk adults. Am J Public Health 2003; 93:2074-8.

3. Rangel MC, Shoenbach VJ, Weigle KA, et al. Racial and ethnic disparities in influenza vaccination among elderly adults. J Gen Intern Med 2005;20:426-31.

4. Linn ST, Guralnik JM, Patel KV. Disparities in influenza vaccine coverage in the United States, 2008. J Am Geriatr Soc 2010; 58:1333-40.

5. Flowers L, Sinclaire S-A. Racial and ethnic disparities in influenza and pneumococcal immunization rates among 
medicare beneficiaries. Washington (DC): AARP Public Policy Institute; 2008.

6. Fiscella K. Commentary - anatomy of racial disparity in influenza vaccination. Health Serv Res 2005;40:539-49.

7. Ethnic origin and visible minorities. Ottawa $(\mathrm{ON})$ : Statistics Canada; 2006.

8. Chui T, Tran K, Maheux H. 2006 Census: immigration in Canada: a portrait of the foreign-born population, 2006 Census: findings. Ottawa $(\mathrm{ON}) ; 2006$.

9. Public Health Agency of Canada. Statement on seasonal trivalent inactivated influenza vaccine (TIV) for 2010-2011. Ottawa (ON): The Agency; 2010. p. 49

10. Public Health Agency of Canada. Publicly funded immunization programs in Canada - routine schedule for infants and children including special programs and catch-up programs (as of March 2011). Ottawa (ON): The Agency; 2011.

11. Canadian Community Health Survey 2000-2009. Ottawa (ON): Statistics Canada.

12. Thomas S, Tremblay S. Interpreting estimates from the redesigned Canadian Community Health Survey (CCHS). Ottawa (ON): Statistics Canada; 2008

13. Thomas W. Combining cycles of the Canadian Community Health Survey. Ottawa (ON): Statistics Canada; 2009.

14. Gagné C, Roberts G, Keown L. Weighted estimation and bootstrap variance estimation for analysing survey data: how to implement in selected software. Ottawa (ON): Statistics Canada; 2011.

15. Bootvar macro user guide. Ottawa (ON): Statistics Canada; 2005.

16. Daniels NA, Gildengorin G, Nguyen T, et al. Influenza and pneumococcal vaccination rates among Vietnamese, Asian, and non-Hispanic white Americans. J Immigr Minor Health 2010; 12:370-6.

17. Setse R, Euler G, Gonzalez-Feliciano A, et al. Influenza vaccination coverage - United States, 2000-2010. MMWR Surveill Summ 2011;60(Suppl):38-41.

18. Bish A, Yardley L, Nicoll A, et al. Factors associated with uptake of vaccination against pandemic influenza: a systematic review. Vaccine 2011;29:6472-84

19. Brien S, Kwong JC, Buckeridge DL. The determinants of 2009 pandemic $\mathrm{A} / \mathrm{H} 1 \mathrm{~N} 1$ influenza vaccination: a systematic review. Vaccine 2012;30:1255-64.

20. Guttmann A, Manuel D, Stukel TA, et al. Immunization coverage among young children of urban immigrant mothers: findings from a universal health care system. Ambul Pediatr 2008;8:205-9.

21. Mixer RE, Jamrozik K, Newsom D. Ethnicity as a correlate of the uptake of the first dose of mumps, measles and rubella vaccine. J Epidemiol Community Health 2007;61:797-801

22. Link MW, Ahluwalia IB, Euler GL, et al. Racial and ethnic disparities in influenza vaccination coverage among adults during the 2004-2005 season. Am J Epidemiol 2006;163:571-8.

23. Logan JL. Disparities in influenza immunization among US adults. J Natl Med Assoc 2009;101:161-6.

24. Van D, McLaws M, Crimmins J, et al. University life and pandemic influenza: attitudes and intended behaviour of staff and students towards pandemic (H1N1) 2009. BMC Public Health 2010;10:130.

25. Martin LM, Leff M, Calonge N, et al. Validation of self-reported chronic conditions and health services in a managed care population. Am J Prev Med 2000;18:215-8.

26. Skull SA, Andrews RM, Byrnes GB, et al. Validity of selfreported influenza and pneumococcal vaccination status among a cohort of hospitalized elderly inpatients. Vaccine 2007;25: 4775-83.

27. Mac Donald R, Baken L, Nelson A, et al. Validation of selfreport of influenza and pneumococcal vaccination status in elderly outpatients. Am J Prev Med 1999;16:173-7.

28. Gilmore J. The 2008 Canadian immigrant labour market: analysis of quality of employment. Ottawa (ON): Statistics Canada; 2008.

Affiliations: From Surveillance and Epidemiology (Quach, Pereira, Heidebrecht, Deeks, Crowcroft), Public Health Ontario, Toronto, Ont.; the Department of Clinical Epidemiology and Biostatistics (Hamid), McMaster University, Hamilton, Ont.; the Dalla Lana School of Public Health (Deeks, Crowcroft, Kwong), University of Toronto; the Department of Laboratory Medicine and Pathobiology (Crowcroft), University of Toronto; the University Health Network (Quan), Toronto, Ont.; the Department of Epidemiology, Biostatistics, and Occupational Health (Brien), McGill University, Montréal, Que.; the Institute for Clinical Evaluative Sciences (Kwong); and the Department of Family and Community Medicine (Kwong), University of Toronto, Toronto Ont.

Contributors: Susan Quach conceived the study, performed the analyses and prepared the first draft of the manuscript. All of the authors interpreted the data, critically revised the manuscript for important intellectual content and approved the final version submitted for publication.

Funding: This study was supported by an operating grant from the Public Health Agency of Canada and the Canadian Institutes of Health Research. The study sponsors had no role in the design of the study, the collection, analysis or interpretation of data, the writing of the report or the decision to submit the article for publication.

Acknowledgements: The authors thank the people at Statistics Canada for their contributions and support, and the Canadian Association for Immunization Research and Evaluation for provided networking assistance.

Vaccine Coverage Theme Group: Julie Bettinger, Stephanie Brien, David Buckeridge, Larry Chambers, Natasha Crowcroft, Lois Crowe, Shelley Deeks, Michael Finkelstein, Maryse Guay, Jemila Hamid, Christine Heidebrecht, Donna Kalailieff, Faron Kolbe, Jeff Kwong, Allison McGeer, Jane Nassif, Jennifer Pereira, Susan Quach, Sherman Quan and Chris Sikora. 